\section{Conocimientos, Opiniones y Hábito de Fumar en Profesores*}

Drago Vrsalović Mihoević**

Aida Kirschbaum Kasten $* * *$

Dada la trascendencia que tienen las actividades educativas en la prevención del hábito de fumar en niños $y$ jóvenes, se realizó un estudio con la finalidad de conocer las tasas de prevalencia, conocimientos y opiniones que tienen los profesores sobre el tabaquismo. Para ello se aplicó un cuestionario a 746 profesores de la ciudad de Osorno, Chile.

Los resultados indicaron una tasa de 42,6\% de maestros fumadores; considerando la especialización, las mayores prevalencias se hallan en las profesoras de Biología y de Educación Física. Se establecen comparaciones con prevalencias de otros paises.

Se determinó que el nivel de conocimientos es independiente del hábito de fumar, en cambio, las opiniones expresadas sobre diversos aspectos vinculados al tabaquismo, están fuertemente asociadas a su hábito. Se concluye la necesidad de realizar una capacitación sistemática a los profesores en servicio y en los Centros de formación de maestros.

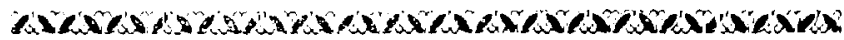

\section{INTRODUCCION}

El tabaquismo es uno de los problemas de Salud Pública relevantes del mundo actual, debido a que es un hábito muy difundido que es capaz de generar diversos tipos de morbilidad, de producir mortalidad e importantes gastos en atención médica. Ya en el año 1970, el Real Colegio Médico de Londres sostenía que las enfermedades asociadas con el mayor hábito de fumar causan tantas muertes que representan el desafio para las acciones preventivas en salud (14). Esta afirmación que se refería a los países desarrollados tiene una mayor trascendencia en la actualidad, debido a que en los paises en desarrollo Cadernos de Saúde Pública, RJ, 7(3): 347-362, jul/set, 1991
*Investigación financiada por la Dirección de Investigación y Bibliotecas del Instituto Profesional de Osorno.

**Departamento de Educación y Ciencias, Instituto Profesional de Osorno, Casilla 933. Osorno, Chile.

***Escuela de Salud Pública, Facultad de Medicina, Universidad de Chile. Santiago, Chile. 
también se están sufriendo las consecuencias de esta costumbre.

El consumo de cigarrillos y sus factores asociados, es un fenómeno necesario de estudiar y profundizar a nivel de la niñez y de la adolescencia, porque es la época en que se comienza a practicar el hábito y por lo tanto, la etapa de la vida prioritaria para desarrollar acciones educativas con énfasis en la prevención.

Estudios realizados en alumnos chilenos de Enseñanza Media, revelaron una prevalencia del hábito de fumar que fluctuó entre el $53 \%$ y el $65 \%(3,8)$, y que el $60 \%$ de los escolares comienzan a los 14 años (21); en Osorno se encontró (D. Vrsalović, observaciones inéditas, 1988) una tasa de 51,5\%, expresando el $45 \%$ de ellos, que comenzaron a fumar antes de los 15 años. En la Enseñanza Básica se han determinado prevalencias de $57,6 \%$ en el año 1982 (7) y de $40,7 \%$ en 1987 (15), ambos en grupos de escuelas de Santiago.

La Organización Mundial de la Salud, dentro de las medidas de lucha antitabáquica, da una gran trascendencia a los aspectos educativos señalando que las escuelas pueden servir como el conducto más importante para un público de suma importancia y plantea el hecho de tener acceso a grupos de jóvenes que no han comenzado todavía a fumar, indicando que los maestros que enseñan materias como Biologia, Educación Física y en algunos paises Religión, son útiles portadores de mensajes educativos contra el hábito de fumar (16).

En base a lo expuesto, resultan fundamentales las acciones que tengan como objetivo evitar que los jóvenes comiencen a fumar, por lo tanto, es indispensable comenzar una educación sobre el tabaquismo en forma sistemática, a nivel escolar. Pero antes de incorporar a las Instituciones Educacionales en la lucha contra este hábito, se debe tener la seguridad de contar con un profesorado debidamente capacitado en este tema y con una clara actitud antitabáquica, ya que el maestro no sólo cumple un papel de instructor, sino también desempeña un importante rol de modelo conductual ante sus alumnos, en cualquier asignatura que desarrolle.

Este trabajo aporta un conjunto de antecedentes que pueden contribuir a la adopción de medidas que permitan mejorar la calidad de la enseñanza sobre esta temática a nivel escolar, e implementar efectivos programas de 
prevención en los cuales tenga una activa participación el profesorado.

\section{MATERIALES Y METODOS}

Para este trabajo se consideró como población en estudio, a todos los profesores de Enseñanza Básica y Media que ejercieron sus funciones durante el segundo semestre de 1986 y el primer semestre de 1987 en la ciudad de Osorno, la cual se encuentra a $900 \mathrm{~km}$ al sur de Santiago y cuenta con una población de 100.000 habitantes.

En Chile, los maestros de Enseñanza Básica (E.B.) ejercen la docencia con niños de 6 a 13 años y los de Enseñanza Media (E.M.), de los 14 hasta 17 años.

El instrumento utilizado para la obtención de los datos fue un cuestionario anónimo, el cual se elaboró con preguntas estructuradas; dicho instrumento contiene preguntas para ser contestadas por todos los encuestados y otras para que las respondieran los profesores según se clasificaran como Fumadores Actuales, Dejaron de Fumar o Nunca Fumaron. Se recogieron datos de 746 profesores, lo que correspondió al $93,7 \%$ de los docentes.

Teniendo presente que la distribución etárea de los profesores es muy diferente según el nivel de enseñanza, se empleó el método de Ajuste de Tasas, tomando como población standard la de Enseñanza Media. Hubo 17 maestros que no indicaron su edad, lo que representó un $2,3 \%$.

El procesamiento de la información se llevó a cabo por computación y para ello se diseñaron los programas necesarios para almacenar y procesar los datos. Para establecer el nivel de asociación entre las variables seleccionadas, se empleó la Prueba de Asociación del Test chi cuadrado, a un nivel de significación del $1 \%$.

Se definió como Fumador al profesor que ha fumado más de 5 cajetillas durante su vida y que a la fecha de la aplicación del cuestionario estaba fumando; a los maestros que han fumado más de 5 cajetillas durante su vida, pero que en esa fecha no fumaban, se les clasificó como Dejaron de Fumar, y los que Nunca Fumaron corresponden a los que habían fumado menos de 5 cajetillas en su vida, o bien, no lo habían hecho nunca (17). 


\section{RESULTADOS}

\section{Prevalencias Total y Específicas}

El cuadro I indica que la tasa de prevalencia del hábito de fumar en los profesores es del $42,6 \%$, siendo más alto en el sexo masculino.

\section{CUADRO I}

Tasas de Prevalencia del Hábito de Fumar por 100 Profesores, según Sexo. Osorno, 1987

\begin{tabular}{lcccccc}
\hline Hábito de & \multicolumn{2}{c}{ Hombres } & \multicolumn{2}{c}{ Mujeres } & \multicolumn{2}{c}{ Total } \\
Fumar & $\mathrm{n}$ & Tasa & $\mathrm{n}$ & Tasa & $\mathrm{n}$ & Tasa \\
\hline Fumadores & 118 & 48,4 & 200 & 39,8 & 318 & 42,6 \\
Dejaron Fumar & 58 & 23,8 & 109 & 21,7 & 167 & 22,4 \\
Nunca Fumaron & 68 & 27,8 & 193 & 38,4 & 261 & 34,9 \\
\hline Total & 244 & 100 & 502 & 100 & 746 & 100 \\
\hline
\end{tabular}

Al desglosarlo por nivel de enseñanza, se aprecia en el cuadro II, que las tasas de los fumadores son notablemente diferentes, alcanzando significación estadística $(\mathrm{p}: 0,01)$. Al calcular la tasa ajustada por edad, los maestros de E.B. aumentan al 46,3\%, acercándose bastante a los docentes de E.M.

\section{CUADRO I}

Tasas de Prevalencia del Hábito de Fumar por 100 Profesores, según Nivel de Enseñanza. Osorno, 1987

\begin{tabular}{lccccccc}
\hline Hábito de & $\begin{array}{r}\text { Enseñanza } \\
\text { Media }\end{array}$ & \multicolumn{2}{c}{$\begin{array}{r}\text { Enseñanza } \\
\text { Basica }\end{array}$} & \multicolumn{2}{c}{ Total } \\
Fumar & $\mathrm{n}$ & Tasa & $\mathrm{n}$ & Tasa & $\mathrm{n}$ & Tasa \\
\hline Fumadores & 162 & 50,8 & 156 & 36,5 & 318 & 42,6 \\
Dejaron Fumar & 63 & 19,7 & 104 & 24,4 & 167 & 22,4 \\
Nunca Fumaron & 94 & 29,5 & 167 & 39,1 & 261 & 34,9 \\
\hline Total & 319 & 100 & 427 & 100 & 746 & 100 \\
\hline
\end{tabular}


Al considerar la variable edad, las tasas que muestra el cuadro III reflejan que el grupo de menor edad es mucho más fumador que los otros grupos.

\section{CUADRO III}

Tasas de Prevalencia del Hábito de Fumar por 100 Profesores, según Grupos de Edad. Osorno, 1987

\begin{tabular}{rrrrrrrr}
\hline Grupos de & \multicolumn{2}{l}{ Fumadores } & \multicolumn{2}{c}{ Dejaron de Fumar } & Nunca Fumaron & Total \\
Edad & $\mathrm{n}$ & Tasa & $\mathrm{n}$ & Tasa & $\mathrm{n}$ & Tasa & $\mathrm{n}$ \\
\hline-35 & 113 & 57,1 & 33 & 16,7 & 52 & 26,3 & 198 \\
35 a 44 & 129 & 42,6 & 66 & 21,7 & 108 & 35,6 & 303 \\
$45 \mathrm{y}+$ & 68 & 29,8 & 65 & 28,5 & 195 & 41,7 & 228 \\
Total & 310 & 42,5 & 164 & 22,5 & 255 & 35,0 & 729 \\
\hline
\end{tabular}

Agrupando las menciones en que desarrollan la docencia los profesores de E.M., el área científica presentó una tasa de $53,2 \%$, en el área humanista son fumadores el $50 \%$ y en la enseñanza técnico-profesional la tasa es de $48,4 \%$.

Para establecer una comparación entre diferentes menciones de los maestros y su hábito de fumar, se seleccionó a los profesores de Biología y Educación Física, en función de que su trabajo pedagógico se vincula con los efectos que produce el cigarrillo en el organismo y como contrapartida, a los docentes de Matemática y Castellano. Las tasas de los fumadores son similares en las 4 especialidades, ya que varía desde un $57,5 \%$ en Biología a $52,3 \%$ en Castellano. Según el sexo, las mujeres presentan las mayores tasas, que son del $69,2 \%$ y del $63,6 \%$ en las profesoras de Biología y Educación Física respectivamente.

\section{Características de los Fumadores}

Los profesores varones de E.M. presentan tasas alrededor del $48 \%$ en todos los grupos de edades, en cambio, las prevalencias de las mujeres varían desde el $61,8 \%$ en las menores de 35 años, al $34,8 \%$ en el último grupo. Los profesores de E.B. disminuyen sus tasas a medida que aumenta la edad, tanto en varones como en damas, 
presentando éstas una prevalencia del $23,9 \%$ en el grupo de mayor edad.

Los maestros de menos de 35 años tienen tasas semejantes en los dos niveles de enseñanza, en cambio, los profesores de E.M. mayores de 45 años son mucho más fumadores que los básicos, los cuales alcanzan sólo el 27,1\%; (cuadro IV).

\section{CUADRO IV}

Tasas de Prevalencia de Fumadores por 100 Profesores, según Nível de Enseñanza y Grupos de Edad. Osorno, 1987

\begin{tabular}{ccccccc}
\hline Grupos de & \multicolumn{2}{c}{ E. Media } & \multicolumn{2}{c}{ E. Basica } & \multicolumn{2}{c}{ Total } \\
Edad & n & Tasa" & n & Tasa* & n & Tasa \\
\hline-35 & 85 & 56,7 & 28 & 58,3 & 113 & 57,1 \\
35 a 44 & 58 & 47,5 & 71 & 39,2 & 129 & 42,6 \\
45 y & 19 & 40,4 & 49 & 27,1 & 68 & 29,8 \\
Total & 162 & 52,3 & 148 & 47,7 & 310 & 42,5 \\
\hline
\end{tabular}

a Tasa calculada sobre el total de profesores por grupo de edad, en cada nivel de enseñanza.

Respecto al número de cigarrillos consumidos diariamente, el $77,7 \%$ de los profesores consume menos de 10 , y el $4,7 \%$ fuma 20 ó más. Se aprecia que los fumadores entre 1 a 9 cigarrillos son diferentes según el sexo, ya que las mujeres lo hacen en el $83 \%$ y los varones en el $68,6 \%$; las diferencias observadas en cuanto al sexo, tienen significación estadística ( $p: 0,01$ ).

Al preguntarles a los maestros el grado de preocupación que tienen por los efectos derivados del consumo de cigarrillos en su salud, el cuadro $V$ muestra que un poco más de un tercio de los profesores expresaron estar preocupados, encontrándose niveles muy parecidos según el nivel de enseñanza. Al considerar el sexo, las damas expresaron una mayor despreocupación, especialmente en la E.M.

$\mathrm{Al}$ averiguar sobre la perspectiva que tienen los maestros de continuar fumando dentro de 5 años, el $38,3 \%$ expresó que con seguridad o probablemente Sí continuarán con el hábito, el 29,2\% manifestó que con seguridad o probablemente No continuarán fumando y el resto contestó que no sabe. 


\section{CUADRO V}

Nivel de Preocupación de Profesores Fumadores por los

Riesgos del Tabaco, según Nivel de Enseñanza y Sexo.

Osomo, 1987

\begin{tabular}{|c|c|c|c|c|c|c|}
\hline \multirow{3}{*}{$\begin{array}{r}\text { Nivel de } \\
\text { Enseñanza } \\
\text { Sexo }\end{array}$} & \multirow{2}{*}{\multicolumn{2}{|c|}{$\begin{array}{l}\text { Muy Preocupados } \\
\text { Y Preacupados }\end{array}$}} & \multirow{2}{*}{\multicolumn{2}{|c|}{$\begin{array}{l}\text { Algo Preocupados } \\
\text { y No Preocupados }\end{array}$}} & \multicolumn{2}{|c|}{ Total } \\
\hline & & & & & \multirow[t]{2}{*}{$\mathrm{n}$} & \multirow[t]{2}{*}{$\%$} \\
\hline & $\mathrm{n}$ & $\%$ & $\mathbf{n}$ & $\%$ & & \\
\hline Enseñanza Básica & 52 & 33,3 & 104 & 66,7 & 156 & 100 \\
\hline Hombres & 15 & 37,5 & 25 & 62,5 & 40 & \\
\hline Mujeres & 37 & 31,9 & 79 & 68,1 & 116 & \\
\hline Enseñanza Media & 58 & 35,8 & 104 & 64,2 & 162 & 100 \\
\hline Hombres & 35 & 44,9 & 43 & 55,1 & 78 & \\
\hline Mujeres & 23 & 27,4 & 61 & 72,6 & 84 & \\
\hline Total & 110 & 34,6 & 208 & 65,4 & 318 & 100 \\
\hline
\end{tabular}

\section{Fuentes de Información}

En relación a las fuentes de información a través de las cuales se informaron los profesores sobre los riesgos del consumo de cigarrillos, se obtuvieron los siguientes resultados: el $60,5 \%$ indicó a los diarios y revistas, el $53,1 \%$ a la televisión, y en revistas técnicas el $12,9 \%$. Otras alternativas fueron marcadas en menor proporción; al contestar este ítem, cada maestro podía contestar hasta 2 opciones.

Al separarlos por sexo, estas tres primeras fuentes indicadas coinciden tanto para las damas como para los varones.

Considerando el nivel de enseñanza, los profesores de E.M. señalaron en primer lugar a los diarios y revistas en un $73,4 \%$, en cambio, los prof. de E.B. indicaron la televisión en un $53,4 \%$, como su principal fuente de información.

\section{Conocimientos sobre Patologías Asociadas al Consumo} de Tabaco

En el cuadro VI se dan a conocer los porcentajes de respuestas correctas, que dieron los profesores sobre 
algunas de las patologías que se encuentran estrechamente vinculadas al consumo de tabaco. Se puede apreciar que el cáncer al pulmón fue el problema de salud que más se indicó, con un $92,4 \%$; los encuestados podían marcar sí, no o no sé.

\section{CUADRO VI}

Porcentagens de Respuestas Correctas de los Profesores sobre Consecuencias del Consumo de Cigarrillos, según Hábito de

Fumar y Sexo. Osorno, 1987

\begin{tabular}{|c|c|c|c|c|c|}
\hline $\begin{array}{r}\text { Consecuencias } \\
\text { Sexo }\end{array}$ & Fumadores & $\begin{array}{l}\text { Dejaron } \\
\text { de Fumar }\end{array}$ & $\begin{array}{l}\text { Nunca } \\
\text { Fumaron }\end{array}$ & $\begin{array}{r}\text { Total } \\
\text { P. Sexo }\end{array}$ & $\begin{array}{r}\text { Total } \\
\text { General }\end{array}$ \\
\hline \multicolumn{6}{|l|}{ Cáncer Pulmón } \\
\hline Masc. & 92,4 & 98,3 & 94,1 & 94,3 & \multirow{2}{*}{92,4} \\
\hline Femen. & 88,0 & 94,5 & 93,3 & 91,4 & \\
\hline \multicolumn{6}{|l|}{ Cáncer Vejiga } \\
\hline Masc. & 12,7 & 12,1 & 10,3 & 11,9 & \multirow[t]{2}{*}{10,2} \\
\hline Femen. & 8,0 & 10,1 & 10,4 & 9,4 & \\
\hline \multicolumn{6}{|l|}{$\begin{array}{r}\text { Menos } \mathrm{O}_{2} \\
\text { Cerebro }\end{array}$} \\
\hline Masc. & 61,0 & 53,4 & 50,0 & 56,1 & \multirow{2}{*}{58,2} \\
\hline Femen. & 56,0 & 59,6 & 62,2 & 59,2 & \\
\hline \multicolumn{6}{|l|}{$\begin{array}{r}\text { Menor peso } \\
\text { RN. }\end{array}$} \\
\hline Masc. & 73,7 & 77,6 & 75,0 & 75,0 & \multirow{2}{*}{79,4} \\
\hline Femen. & 82,5 & 81,7 & 80,3 & 81,5 & \\
\hline
\end{tabular}

Referente a úlcera al estómago, hubo un $46,9 \%$ de respuestas correctas, variando el porcentaje entre un $51,4 \%$ de las mujeres que dejaron de fumar y el $38,2 \%$ de los hombres que nunca fumaron.

Las respuestas correctas disminuyeron al $26,9 \%$ cuando se les preguntó sobre la úlcera duodenal, correspondiendo el mayor porcentaje a las mujeres que nunca fumaron con el $30,6 \%$ y el menor a los hombres que dejaron de fumar con el $20,7 \%$.

El 57,6\% contestó que el endurecimiento de las arterias 
es una problemática de salud asociada al consumo de cigarrillos; los varones que dejaron de fumar lo indicaron en el $67,2 \%$ y las damas fumadoras en el $53,5 \%$.

El test de asociación se aplicó por separado a cada pregunta sobre las consecuencias del hábito, ya sea considerando al total de los profesores, como desglosándolos por sexo, no encontrándose significación estadistica en ninguna de ellas.

\section{Opiniones sobre Diversos Aspectos Vinculados al Tabaquismo}

Se le plantearon a los docentes varias situaciones relacionadas con el tabaquismo, a las cuales ellos debían responder con sí, no o nó sé; en el cuadro VII se muestran los porcentajes de respuestas afirmativas.

Se aprecia que casi las tres cuartas partes de los prof. expresaron estar de acuerdo en que los profesionales de la salud no deberían fumar en público; hay significación estadística para el sexo femenino (p: 0,01). En cambio, cuando se les consultó si los profesores deberian dejar de fumar en público, el porcentaje disminuyó al 55,6\%; llama particularmente la atención la baja proporción de aceptación expresada por los fumadores varones. Hay una alta asociación para ambos sexos (p: 0,01).

El Ministerio de Educación de Chile, en mayo de 1981 sugirió, a través de una circular enviada a todos los establecimientos educacionales, que los profesores se abstengan de fumar durante el desarrollo de sus clases y en general, durante el cumplimiento de sus obligaciones frente a alumnos y apoderados. Al preguntarles su opinión al respecto, casi el $90 \%$ está de acuerdo con no fumar en la sala de clases, pero en relación a no fumar en patios, pasillos y delante de apoderados, el porcentaje de respuestas positivas disminuyó al $68,8 \%$; para ambas respuestas, se halló significación estadística solamente para el sexo femenino ( $p: 0,01$ ).

Al preguntarles si estarían de acuerdo en que se prohibiera totalmente la propaganda sobre el cigarrillo, el $60,3 \%$ expresó una opinión positiva, variando entre el $69,7 \%$ de las mujeres que dejaron de fumar y el $50,5 \%$ de las profesoras fumadoras. Prohibir completamente la venta de cigarrillos tuvo el apoyo de sólo el $28 \%$ del profesorado; en las mujeres que dejaron de fumar el porcentaje fue de 
$36,7 \%$, pero en los hombres fumadores fue del $17,8 \%$. En las dos respuestas hubo significación estadística en el sexo femenino (p: 0,01).

\section{CUADRO VII}

Porcentajes de Opiniones Afirmativas de los Profesores sobre Aspectos Vinculados al Tabaquismo, según Hábito de Fumar y

Sexo. Osomo, 1987

\begin{tabular}{|c|c|c|c|c|c|}
\hline $\begin{array}{r}\text { Opiniones } \\
\text { Sexo }\end{array}$ & Fumadores & $\begin{array}{r}\text { Dejaron } \\
\text { de Fumar }\end{array}$ & $\begin{array}{r}\text { Nunca } \\
\text { Fumaron }\end{array}$ & $\begin{array}{r}\text { Total } \\
\text { P. Sexo }\end{array}$ & $\begin{array}{r}\text { Total } \\
\text { General }\end{array}$ \\
\hline \multicolumn{6}{|l|}{$\begin{array}{l}\text { Prof. de salud } \\
\text { s/fumar } \\
\text { público }\end{array}$} \\
\hline Masc. & 60,2 & 75,9 & 70,6 & 66,8 & \\
\hline Femen. & 64,0 & 86,2 & 81,3 & 75,5 & 72,7 \\
\hline \multicolumn{6}{|l|}{ Profesores sin } \\
\hline \multicolumn{6}{|l|}{ fumar publico } \\
\hline Masc. & 30,5 & 65,5 & 54,4 & 45,5 & \\
\hline Femen. & 44,5 & 66,1 & 74,1 & 60,6 & 55,6 \\
\hline \multicolumn{6}{|l|}{$\begin{array}{l}\text { Prohibido } \\
\text { fumar }\end{array}$} \\
\hline \multicolumn{6}{|l|}{ en clases } \\
\hline Masc. & 77,1 & 82,8 & 88,2 & 81,6 & \\
\hline Femen. & 89,0 & 96,3 & 96,9 & 93,6 & 89,7 \\
\hline \multicolumn{6}{|l|}{$\begin{array}{l}\text { Prohibido } \\
\text { fumar }\end{array}$} \\
\hline \multicolumn{6}{|l|}{ en patios } \\
\hline Masc. & 50,8 & 69,0 & 66,2 & 59,4 & \\
\hline Femen. & 64,0 & 76,1 & 81,3 & 73,3 & 68,8 \\
\hline
\end{tabular}

El grado de acuerdo al planteamiento de prohibir por ley la venta de cigarrillos a menores de 18 años, aumentó al $65,4 \%$, variando entre el $76,2 \%$ de las mujeres que nunca fumaron y el $54,2 \%$ de los varones fumadores. 


\section{DISCUSION}

Los variados efectos que produce el consumo de tabaco en la salud de las personas es un asunto tan claramente expuesto en numerosas publicaciones, que prácticamente no pueden ser discutidos, salvo que haya fuertes intereses económicos de por medio.

Actualmente la lucha antitabáquica debe poner énfasis en acciones educativas que permitan lograr en la sociedad, por una parte, la adopción de conductas positivas que fomenten y conserven una buena salud, y por otra, el abandono de conductas negativas como lo es el hábito de fumar.

La información obtenida en este estudio, permite comparar la prevalencia del hábito de fumar en Osorno con dos trabajos realizados en Santiago, cuyos resultados son similares, de $42,6 \%$ (19) y de $40,2 \%$ (20), siendo un poco mayor en los hombres. En otros paises como Estonia y Rumania, los varones presentaron tasas de $40,3 \%$ y $44 \%$, y las damas de $11,1 \%$ y $27,8 \%$ respectivamente (18); en Sud-Africa el año 1978 se comprobó que el $81,6 \%$ de los profesores varones eran fumadores (9). En cambio, Londres y Estados Unidos tenían tasas de un $21 \%$ de fumadores hace más de una década $(2,10)$ y en el norte de Inglaterra durante 1982, se encontró un $18 \%$ de maestros con el hábito (6). Se observa entonces una notoria diferencia entre los fumadores de los países, según el grado de desarrollo que presentan.

La elevada prevalencia de fumadores entre los profesores de Osorno, es un poco superior a la tasa de la población general de la capital del país, que en 1984 alcanzó al 41,6\% (12), pero es claramente superior a las tasas de once de las doce ciudades estudiadas el año 85 (13), lo cual indica que estos profesionales no han tomado todavía conciencia del problema.

El nivel de enseñanza en que se desempeñan los maestros no tiene influencia importante en el hábito, ya que al calcular las tasas ajustadas por la edad, los valores tienden a acercarse bastante. Llama la atención la mayor prevalencia de fumadores, en algunas áreas de especialización cuyas temáticas de enseñanza se relacionan con materias de salud y específicamente con el tabaquismo, como es el caso de las profesoras de Biología y Educación Física, que superan el $63 \%$ de fumadoras.

Una vez más se aprecia el efecto de cohorte, en que los grupos de mayor edad fuman menos que los jóvenes, ya sea Cadernos de Saúde Pública, RJ, 7(3): 347-362, jul/set, 1991 
considerando el total de profesores, o bien al desglosarlos por nivel de enseñanza y por sexo. En cuanto al número de cigarrillos diarios, tres de cada cuatro maestros fumadores consumen menos de media cajetilla, situación inversa a la que se encontró en el norte de Inglaterra (6) y en Estonia (18).

Las altas prevalencias de fumadores se relacionan con los altos porcentajes de despreocupación por los efectos patológicos del tabaco, situación que también se presenta en otros estudios $(12,13)$. Por otra parte, menos de un tercio de los profesores expresan que probablemente dentro de 5 años no continuarán con el hábito, lo cual no da una buena perspectiva a mediano plazo, salvo que se introduzca un factor educativo que modifique dicha predicción.

Las principales fuentes de información expresadas por los docentes sobre los riesgos del consumo de cigarrillos, corresponden a los medios de comunicación de masas, es decir, las mismas fuentes a que tiene acceso la población del país, indicando que falta una preparación más directa a este grupo de profesionales. Es necesario tener presente, que los individuos expuestos a los medios de comunicación social se informan sobre el problema del tabaco, pero a la vez, son estimulados hacia el consumo de cigarrillos por medio de una publicidad engañosa que relaciona este hábito con la sexualidad, juventud, ambientes agradables, etc.

Referente a los conocimientos que poseen, se puede apreciar que los resultados son regulares, ya que sólo frente a dos preguntas las respuestas superan el $70 \%$ de rendimiento. La relación entre cáncer al pulmón y consumo de tabaco ha sido promocionada intensamente no sólo en Chile sino también en otros países, como por ejemplo a través de las frases de advertencia en las cajetillas de cigarrillos (5), además de la natural vinculación entre aspirar el humo y su conducción a los pulmones, razón por la cual, es normal que esta patología se conozca muy bien. Los bajos porcentajes frente a otras morbilidades, indican nuevamente que los maestros no han sido capacitados en forma sistemática.

Las opiniones de los docentes sobre diversos aspectos relacionados al tabaquismo están asociadas a su hábito de fumar, especialmente en el sexo femenino; también se puede apreciar que sus opiniones no manifiestan una clara posición antitabáquica, lo cual se ve reflejado en las respuestas sobre la propaganda y venta de cigarrillos. 
El hecho de que un alto número de fumadores esté cursando carreras pedagógicas en la Educación Superior chilena (4) (Drago Vrsalović, observaciones inéditas, 1987), sugiere la necesidad de que estos Centros estudien la posibilidad de incorporar en los planes de estudio, contenidos que permitan a los alumnos adquirir una visión globalizada sobre este tema y los prepare para aplicar adecuadas estrategias metodológicas de prevención primaria del hábito de fumar, en su futura labor como maestro.

Hay experiencias educativas que indican la factibilidad de influir positivamente sobre el hábito de fumar en adolescentes $(22,1)$, pero no se realizó un seguimiento de algunos años para evaluar así la consistencia de los cambios. Es importante tener presente en los programas educativos que se diseñen, la conveniencia de incorporar un enfoque sicosocial del hábito (11), donde las patologías derivadas del consumo de tabaco se desarrollen, pero no como elemento principal, ya que las acciones preventivas del tabaquismo en los jóvenes que se basan exclusivamente en informar sobre dichos efectos, carecen de impacto en términos de evitar la adquisición del hábito de fumar.

Una buena formación de los profesores debiera permitir la reducción de sus propias tasas de prevalencia, y a la vez, habilitarlos para llevar a cabo un efecto multiplicador, que influirá en miles de niños y jóvenes que comprenderán que lo natural del hombre es no fumar.

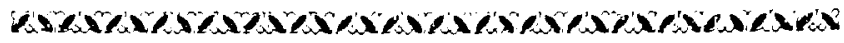

Due to the importance that educational activities have in preventing the habit of smoking in boys and young men, a research was carried out in order to know the rates of predominance, knowledge and opinions teachers have on smoking. For this purpose, an enquiry was applied to 746 teachers from Osorno, Chile.

The results showed a rate of $42.6 \%$ of smokers; considering specialization, the major predominance is found in woman teachers of Biology and Physical Education. Comparisons with predominance in other countries are established. It was determined that the level of knowledge is independent from the habit of smoking; on the contrary, opinions about different aspects related to smoking, are

Cadernos de Saúde Pública, RJ, 7(3): 347-362, jul/set, 1991 
closely connected to the habit.

We conclude that it is necessary to carry on a systematic training with in service teachers and specially in

Teacher's Training Colleges.

\section{NOTA}

Agradecemos al Sr. Ara Pinilla Palma, por su colaboración al diseñar los programas computacionales necesarios para el manejo de los datos.

\section{REFERENCIAS BIBLIOGRAFICAS}

1 - BEHN, B. V. Una estrategia de prevención primaria del tabaquismo en escolares. Archivos de Investigación, Instituto Profesional de Chillán. 3 (1): 7-10, 1985.

2 - BEWLEY, B., JOHNSON, M. y BANKS, M. Teachers' smoking. Journal of Epidemiology and Community Health 33 (3): 219-222, 1979.

3 - CABRERA, R.F., SALOMÓN, R.C., LÓPEZ, B.I. y VIDAL, O.R. El hábito de fumar en estudiantes de Educación Media, en Santiago de Chile. Boletín de la Oficina Sanitaria Panamericana 93 (6): 533-538, 1982.

4 - CRUZ, E., LISBOA, C., AGUIRRE, C., PERTUZE, J. Estudio longitudinal del hábito de fumar en estudiantes de la Universidad Católica. Revista Médica de Chile 113: 1231-1239, 1985.

5 - CHANDLER, U.W. Tabaco: alzar la voz no es suficiente. Foro Mundial de la Salud 7 (3): 235-242, 1986.

6 - CHARLTON, A. Teachers' smoking habits. Community Medicine 6 (4): 273-80, 1984.

7 - CHILE, Ministerio de Salud, Depto. de Programación. "Diagnóstico de Tabaquismo en Chile". Santiago, 1985. Documento de trabajo.

8 - FLORENZANO, U.R., MADRID, V.V., MARTINI, Y.A., SALAZAR, R.M., MARTELLI, L.E. Prevalencia y caracteristicas del consumo de algunas sustancias químicas en estudiantes de Enseñanza Media. Revista Médica de Chile 109: 1051-1059, 1981.

9 - GRIFFITHS, M., KOAPENG, J. Smoking habits of black schoolteachers. Suid-Afrikaanse Mediese Tydskrif 58 (20): 793, 1980.

10 - JAMES, Wg. A study of public school teachers' cigarette smoking attitudes and habits their role in smoking education. Abstract 79-0322, WHO, 1979. 
11 - MARTINEZ, O.L., MOLINA, B.O., MORALES, C.M., MOYA, R.C., PAREDES, M.R. Factores sico-sociales relacionados con el hábito de fumar. Revista Médica de Chile 115: 167-171, 1987.

12 - MEDINA, E., PASCUAL, J., CUMSILLE, F., ALEGRIA, A., GUTIÉRREZ, L., GUTIÉRREZ, G., CORVALÁN, L., BIRON, M., BUHLER, J., CABRERA, V., LARRAÑAGA, J. Encuesta de tabaquismo en la población general de Santiago. Revista Médica de Chile 114 (3); 257-262, 1986.

13 - MEDINA, E., BAHAMONDES, A., ESPINOZA, A., FERNANDEZ, M., FORRAY, B., JERIA, C., KONDRAK, M. Tabaquismo en 12 ciudades chilenas. Cuadernos Médico Sociales 28 (2): 76-82, 1987.

14 - NEGHME, R.A. El tabaquismo epidémico y la salud. Trabajo presentado en: Conferencias Médicas Lat inoamericanas, Santiago, Chile, 20 al 24 de octubre de 1981.

15 - OLIVARI, V.F., DE LA FUENTE, M.M., LOPEZ, L.I. Consumo de tabaco en escolares de $5^{\circ}$ a $8^{\circ}$ Básico de la comuna de Conchali, Santiago. En: Octavas Jornadas Chilenas de Salud Pública. Santiago, Escuela de Salud Pública, Universidad de Chile, 1988, pp. 292-294.

16 - Organización Mundial de la Salud. Estrategias contra el tabaquismo en los paises en desarrollo. Ginebra, 1983. Serie de Informes Técnicos № 695.

17 - Organización Panamericana de la Salud. Encuesta sobre las características del hábito de fumar en América Latina. Washington D.C., 1977. Publicación Científica № 337.

18 - RAUDSEPP, J., RAHU, M. Smoking among Schoolteachers in Estonia 1980. Scandinavian Journal of Social Medicine 12 (1): 49-53, 1984.

19 - RIVERA, V. S., NAVARRO, B.C., TOBAR, B.M., BAEZ, C.D., FERREIRA, C.H., RODRIGUEZ, J.A., RUBIO, L.E., NAVARRO, V.R. El hábito de fumar: $i$ Existe en los profesores?. En: Quintas Jornadas Nacionales Multidisciplinarias de Educación para la salud. Santiago, Ministerio da Salud, 1987, pp. 33-37.

20 - ROMEU, F.C., VIDAL, O.R., DIAZ, T.G. Caracteristicas relacionadas con el hábito de fumar en docentes de Educación Básica y Media, año 1986, comuna Quinta Normal. En: Quintas Jornadas Nac. Multidisciplinarias de Educación para la Salud. Santiago, Ministerio de Salud, 1987, pp. 147-149.

21 - SALAS, P.I., MACKENNA, H.A., MANRIQUEZ, L.M., MARTINEZ, V.M., MOLINA, G.A., MONTALVA, H.S., NIEME, B.E., O'RYAN, G.F., PESERTI, G.L., PETERS, A.G., RAMOS, V.E. Prevalencia de tabaquismo en alumnos de Enseñanza Media de la 


\section{PESQUISA}

comuna de Providencia y Las Condes. Revista Médica de Chile 108 (5): 453-461, 1980.

22 - SALAS, D.I., REPETTO, M.A., VENTURINI, G.G., PALMA, C.C. Eficacia de un programa educativo antitabáquico en jóvenes. En: Segundas Jornadas Chilenas de Salud Pública. Santiago, Escuela de Salud Pública, U. de Chile, 1982, pp. 74-75. 